\title{
Heterogeneous neuronal activity in the lateral habenula after short- and long-term cocaine self-administration in rats
}

\author{
Ping Gao, ${ }^{1,2}$ Henk J. Groenewegen, ${ }^{1,2}$ Louk J. M. J. Vanderschuren ${ }^{3}$ and Pieter Voorn ${ }^{1,2}$ (DD \\ ${ }^{1}$ Department of Anatomy and Neurosciences, VU University Medical Center, de Boelelaan 1108, 1081 HZ Amsterdam, The \\ Netherlands \\ ${ }^{2}$ Neuroscience Campus Amsterdam, Amsterdam, The Netherlands \\ ${ }^{3}$ Division of Behavioural Neuroscience, Department of Animals in Science and Society, Faculty of Veterinary Medicine, Utrecht \\ University, Utrecht, The Netherlands
}

Keywords: c-fos, drug addiction, GAD65, immediate early gene, RMTg

\begin{abstract}
Cocaine addiction is thought to be the result of drug-induced functional changes in a neural network implicated in emotions, learning and cognitive control. Recent studies have implicated the lateral habenula (LHb) in drug-directed behavior, especially its aversive aspects. Limited cocaine exposure has been shown to alter neuronal activity in the LHb, but the impact of long-term drug exposure on habenula function has not been determined. Therefore, using $c$-fos as a marker, we here examined neuronal activity in $\mathrm{LHb}$ in rats that self-administered cocaine for either 10 or 60 days. Both the density of labeled cells and the cellular labeling intensity were measured in the lateral (LHbL) and medial (LHbM) parts of LHb. After 10 days of cocaine self-administration, both the density and intensity of $c$-fos-positive cells were significantly increased in LHbL, but not LHbM, while after 60 days, an increased density (but not intensity) of labeled neurons in both LHbL and LHbM was observed. Most $c$-fos-labeled neurons were glutamatergic. In addition, we found increased GAD65 expression after 10 but not 60 days of cocaine self-administration in the rostral mesencephalic tegmental nucleus. These data shed light on the complex temporal dynamics by which cocaine selfadministration alters activity in LHb circuitry, which may play an important role in the descent to compulsive drug use as a result of prolonged cocaine-taking experience.
\end{abstract}

\section{Introduction}

Drug addiction is a chronic relapsing disorder characterized by loss of control over drug intake (American Psychiatric Association, 2013). Addiction to substances of abuse is thought to be caused by functional changes in a neural network implicated in emotions, learning and cognitive control (Robinson \& Berridge, 2003; Goldstein \& Volkow, 2011; Koob, 2013; Piazza \& DerocheGamonet, 2013; Volkow \& Morales, 2015; Everitt \& Robbins, 2016) as a result of prolonged exposure to drugs (Deroche-Gamonet et al., 2004; Vanderschuren \& Everitt, 2004; Pelloux et al., 2007; Jonkman et al., 2012; Vanderschuren \& Ahmed, 2013; Limpens

Correspondence: Pieter Voorn, as above.

E-mail: p.voorn@vumc.nl

Received 7 June 2017, revised 6 October 2017, accepted 1 November 2017

Edited by Michel Barrot

Reviewed by Salvatore Lecca, University of Lausanne, Switzerland; and Lucas Lecourtier, University of Strasbourg, France

The associated peer review process communications can be found in the online version of this article. et al., 2014). Brain structures widely implicated in addiction include the prefrontal cortex, striatum, amygdala and the ventral tegmental area (VTA) (Jentsch \& Taylor, 1999; Feil et al., 2010; Koob \& Volkow, 2010; Hearing et al., 2012; Everitt \& Robbins, 2016; Oliva \& Wanat, 2016). More recently, studies have begun to investigate the role of the lateral habenula ( $\mathrm{LHb})$ in addictive behavior, and it has been suggested that this structure is important for the aversive aspects of drug intake (Jhou et al., 2013; Meye et al., 2015; Shelton et al., 2016).

The LHb is a crucial node in the circuitry that connects the limbic basal forebrain with the monoaminergic cell groups in the mesencephalon, via which the ascending midbrain dopamine and serotonin projections to the cerebral cortex, basal ganglia and other basal forebrain regions can be modulated (Herkenham \& Nauta, 1979; Araki et al., 1988; Ji \& Shepard, 2007; Matsumoto \& Hikosaka, 2007; Hikosaka et al., 2008; Jhou et al., 2009b). Based on its structure and its afferent and efferent connections, it can be subdivided into a medial part (LHbM) and a lateral part (LHbL) (Herkenham \& Nauta, 1979) that display a complex subnuclear organization (Andres et al., 1999; Geisler et al., 2003). The medial part collects more limbic-related inputs whereas the lateral part is more related to 
basal ganglia (Herkenham \& Nauta, 1977; Kowski et al., 2008). Importantly, LHb can exercise control over dopaminergic neurons, which have been widely implicated in addictive behavior (Robinson \& Berridge, 2003; Koob, 2013; Volkow \& Morales, 2015; Everitt \& Robbins, 2016). LHb exerts its influence via direct efferents from its medial part to VTA, and indirectly by way of projections from its lateral part to the rostral mesencephalic tegmental nucleus (RMTg), which contains GABAergic neurons that, in turn, contact the dopaminergic neurons of VTA (Jhou et al., 2009a; Kaufling et al., 2009; Omelchenko et al., 2009; Brinschwitz et al., 2010; Barrot et al., 2012).

Immediate early gene (IEG) studies have reported effects of shortterm exposure to cocaine on LHb function. It is noteworthy that these cellular responses were far from homogeneous, in that they strongly differed between the lateral and medial compartments of LHb (Wirtshafter et al., 1994; Zhang et al., 2005; Kowski et al., 2009; Zahm et al., 2010). However, little is known about the effects of prolonged drug exposure, that is, long-term cocaine self-administration, on neuronal activation in LHb. As prolonged drug use is a critical factor in the precipitation of addiction, we here examined c-fos expression in the $\mathrm{LHb}$ in animals that experienced 10 days (short-term) or 60 days (long-term) of cocaine self-administration. We compared these cellular activity responses to those after shortor long-term self-administration of the natural reinforcer sucrose. In view of the LHb's complex organization and its considerable length in the sagittal plane, we studied the lateral and medial subregions of LHb throughout its rostrocaudal extent. Furthermore, to establish possible consequences of changes in habenular neuronal activation for regulation of VTA function, we assessed GABAergic neuronal activity in the RMTg by quantifying cellular levels of GAD mRNA. We expected that short-term cocaine self-administration would lead to activation of glutamatergic neurons in the LHb based on the aversive effects of cocaine after limited exposure to the drug (Jhou et al., 2013; Meye et al., 2015; Shelton et al., 2016). For this reason, experiments included double-labeling procedures allowing us to establish a possible glutamatergic identity of the $c$-fos-expressing neurons. Whether after prolonged exposure (60 days), there would be (further) changes in the strength of activation or the pattern of activated neurons is subject of this study.

\section{Materials and methods}

\section{Animals}

Male Wistar rats (Charles River, Sulzfeld, Germany) weighing 320$380 \mathrm{~g}$ were housed individually in Macrolon cages $(L=40 \mathrm{~cm}$, $W=25 \mathrm{~cm}, H=18 \mathrm{~cm})$ under controlled conditions $\left(20-21^{\circ} \mathrm{C}\right.$, $55 \pm 15 \%$ relative humidity) and reversed $12 \mathrm{~h}$ light-dark cycle (lights on at $19 \mathrm{~h}$ ). Each subject received $20 \mathrm{~g}$ laboratory chow (SDS Ltd, UK) per day and free access to water, which was sufficient to maintain body weight and growth. All experiments were approved by the Animal Ethics Committee of Utrecht University and were conducted in agreement with Dutch laws (Wet op de Dierproeven, 1996) and European regulations (Guideline 86/609/EEC). The new EU directives from 2010 have been implemented into Dutch law in 2014. The present experiments were initiated under the old (1996) guidelines and these are valid until January 1, 2018.

\section{Apparatus}

Subjects were trained and tested in operant conditioning chambers $(L=29.5 \mathrm{~cm}, \quad W=32.5 \mathrm{~cm}, \quad H=23.5 \mathrm{~cm} ; \quad$ Med Associates,
Georgia, VT, USA). The chambers were placed in light- and soundattenuating cubicles equipped with a ventilation fan. Each chamber was equipped with two $4.8 \mathrm{~cm}$ wide retractable levers, placed $11.7 \mathrm{~cm}$ apart and $6.0 \mathrm{~cm}$ from the grid floor. A cue light $(28 \mathrm{~V}$, $100 \mathrm{~mA}$ ) was present above each active lever, and a white house light $(28 \mathrm{~V}, 100 \mathrm{~mA})$ was located on the opposite wall. Sucrose pellets (45 mg, formula F; Research Diets, New Brunswick, NJ, USA) were delivered at the wall opposite to the levers via a dispenser. Cocaine infusions were controlled by an infusion pump placed on top of the cubicles. During the cocaine self-administration sessions, polyethylene tubing ran from the syringe placed in the infusion pump via a swivel to the cannula on the animals' back. In the operant chamber, tubing was shielded with a metal spring. Experimental events and data recording were controlled by procedures written in MedState Notation using MED-PC for Windows.

\section{Surgery}

Rats in the cocaine self-administration group were anaesthetized with ketamine hydrochloride $(0.075 \mathrm{mg} / \mathrm{kg}$, i.m. $)$ and medetomidine $(0.40 \mathrm{mg} / \mathrm{kg}$, s.c. $)$ and supplemented with ketamine as needed. A single catheter was implanted in the right jugular vein aimed at the left vena cava. Catheters (Camcaths, Cambridge, UK) consisted of a $22 \mathrm{~g}$ cannula attached to silastic tubing (0.012 ID) and fixed to nylon mesh. The mesh end of the catheter was sutured subcutaneously (s.c.) on the dorsum. Carprofen $(50 \mathrm{mg} / \mathrm{kg}$, s.c.) was administrated once before and twice after surgery. Gentamycin $(50 \mathrm{mg} / \mathrm{kg}$, s.c.) was administered before surgery and for 5 days post-surgery. Animals were allowed 10 days to recover from surgery.

\section{Cocaine and sucrose self-administration procedures}

Rats were trained to self-administer cocaine under a fixed ratio-1 (FR-1) schedule of reinforcement, as previously published (Veeneman et al., 2012; Gao et al., 2017). During the self-administration sessions, two levers were present, an active lever and an inactive lever. The left or right position of the active and inactive levers was counterbalanced for individual animals. Pressing the active lever resulted in the infusion of $0.25 \mathrm{mg}$ cocaine in $0.1 \mathrm{~mL}$ saline over $5.6 \mathrm{~s}$, retraction of the levers and switching off of the house light. During the infusion, a cue light above the lever was switched on, followed by a $20 \mathrm{~s}$ time-out period after which the levers were reintroduced and the house light illuminated. The time-out period was changed to $3 \mathrm{~min}$ after five training sessions to increase the session length. The session ended after $2 \mathrm{~h}$ or if animals had obtained 40 cocaine infusions, whichever occurred first. Responding on the inactive lever had no programmed consequences, but was recorded to assess general levels of activity. After each self-administration session, intravenous catheters were flushed with a gentamycin-heparinsaline solution to maintain the patency of the catheters.

The training procedure for the rats in the sucrose group was similar to that for cocaine self-administration, with the following exception - each response on the active lever resulted in presentation of a sucrose pellet. Subjects in the control group were also exposed to the self-administration box. Each response on the active lever resulted in illumination of the cue light for $5.6 \mathrm{~s}$. With respect to the numbers of animals in experimental groups, in the 10 days experiment, $n=5$ in control, $n=5$ in sucrose, $n=6$ in cocaine groups; in the 60 days experiment, $n=6$ in control, $n=6$ in sucrose, $n=6$ in cocaine groups. For $c$-fos in situ hybridization (ISH), three animals were excluded (one control in the 10 days experiment for both LHbL and LHbM, one control in the 60 days 
experiment for $\mathrm{LHbL}$ and one cocaine animal in the 10 days experiment for LHbM), in all cases due to damage of tissue sections. For GAD65 mRNA ISH, $n=4$ in control, $n=6$ in cocaine groups for both 10 and 60 days experiments.

\section{Tissue dissection}

After the last training session, rats were moved back to their home cage and decapitated after $30 \mathrm{~min}$. Upon approval of the Utrecht Animal Ethical Committee, animals were terminated without prior anesthesia to avoid unwanted effects on IEG expression. Brains were quickly removed and immediately frozen in cold isopentane, then stored at $-80{ }^{\circ} \mathrm{C}$. Fourteen micrometer thick coronal sections were cut at $-25{ }^{\circ} \mathrm{C}$ in a cryostat (Leica CM 1950) and mounted on SuperFrost ${ }^{\circledR}$ Plus glass slides (Menzel-Gläser, Braunschweig, Germany) and stored at $-80{ }^{\circ} \mathrm{C}$.

\section{Probe generation}

Probes for $c$-fos and vGluT2 ISH were designed using PRIMER 3 software. Each primer contained either a T7 or T3 RNA polymerase promoter sequence. A mixture of four different $v G l u T 2$ probes with similar length was used for detecting $v G l u T 2$ mRNA signal. The full sequences of the primers are as follows: c-fos: sense 5- GTAATA CGACTCACTATAGGGTCACCCTGCCTCTTCTCAAT -3 and antisense 5 - AATTAACCCTCACTAAAGGGCACAGCCTGGTGT GTTTCAC -3’, vGluT2 (1): sense 5`- AATTAACCCTCACTAAAG GG CAACTCACAGCCTTGCTGAA -3` and antisense 5`- GTAA TACGACTCACTATAGGGCAGAAGAACGACCCGTGAAT -3’, vGluT2 (2): sense 5`AATTAACCCTCACTAAAGGGATGCCCC TAGCTGGTATCCT -3` and antisense 5`- GTAATACGACTCAC TATAGGGAGCCAACAACCAGAAGCAGT -3`, vGluT2 (3): sense 5 - AATTAACCCTCACTAAAGGGACAAGTCCCGTGAAGAAT GG $-3{ }^{`}$ and antisense 5`- GTAATACGACTCACTATAGGGG AATGGCCTGAATGGAAATG -3’, vGluT2 (4): sense 5` - AATT AACCCTCACTAAAGGGTCAATGAAATCCAACGTCCA -3` and antisense $5^{\prime}$ - GTAATACGACTCACTATAGGGGCAGGTTTAT GCTTCGCACT -3`. Probe generation was started with a total RNA extraction, followed by a cDNA synthesis procedure (Applied Biosystems, Branchburg, NJ, USA). Specific DNA fragments were produced using Phusion ${ }^{\circledR}$ High-Fidelity PCR Kit (New England Biolabs, Germany), and the PCR conditions were $98{ }^{\circ} \mathrm{C}$ for $20 \mathrm{~s}$, $60{ }^{\circ} \mathrm{C}$ for $40 \mathrm{~s}, 72{ }^{\circ} \mathrm{C}$ for $60 \mathrm{~s}$ and a final step at $72{ }^{\circ} \mathrm{C}$ for $10 \mathrm{~min}$. For GAD65 ISH, pBluescript plasmid that contained $2.3 \mathrm{~kb}$ GAD65 DNA and T7 or T3 sequence was kindly supplied by N. Tillakaratne (University of California Los Angeles) (Erlander et al., 1991). The insert DNA in each clone was amplified by PCR with T7 and T3 primers. PCR conditions were $93{ }^{\circ} \mathrm{C}$ for $60 \mathrm{~s}, 55{ }^{\circ} \mathrm{C}$ for $60 \mathrm{~s}$, $72{ }^{\circ} \mathrm{C}$ for $60 \mathrm{~s}$ and a final step at $72{ }^{\circ} \mathrm{C}$ for $10 \mathrm{~min}$. All probes $(c$ fos, vGluT2, GAD65) were produced using MAXIscript ${ }^{\circledR} \mathrm{T} 3 / \mathrm{T} 7$ In vitro Transcription Kit (Ambion Inc, Austin, TX, USA). C-fos and GAD65 probes were labeled by incorporating digoxigenin-labeled UTP, and vGluT2 probes were labeled by Fluorescein-12-UTP (Roche Diagnostics GmbH, Mannheim, Germany).

\section{In situ hybridization}

For c-fos and GAD65 ISH, coronal sections were fixed in $4 \%$ paraformaldehyde, acetylated using acetic anhydride $(0.25 \%$ acetic anhydride in $1.5 \%$ triethanolamine buffer), delipidated and dehydrated. Digoxigenin-labeled RNA probes (5 ng/section) were applied at $60{ }^{\circ} \mathrm{C}$ in a humid chamber to hybridize for $16-20 \mathrm{~h}$.
Post-hybridization stringency washing was $1 \times$ saline-sodium citrate (SSC) at $60{ }^{\circ} \mathrm{C}(30 \mathrm{~min} \times 2)$, followed by a RNase A treatment in $2 \times \mathrm{SSC}$ buffer at $37{ }^{\circ} \mathrm{C}$ for $15 \mathrm{~min}$. After another washing in $1 \times \mathrm{SSC}$ at $60{ }^{\circ} \mathrm{C}(30 \mathrm{~min})$, sections were incubated for $1 \mathrm{~h}$ in $1 \times \mathrm{SSC}$ at room temperature. Subsequently, sections were exposed to blocking solution (1.212\% TRIS [2-Amino-2(hydroxymethyl)propane-1,3-diol], $0.876 \% \quad \mathrm{NaCl}, \quad 1 \%$ blocking powder; Roche Diagnostics $\mathrm{GmbH}$ ) for $1 \mathrm{~h}$ at room temperature and incubated with an anti-digoxigenin antibody conjugated with alkaline phosphatase $(1: 2500)$ (Roche Diagnostics $\mathrm{GmbH}$ ) at $4{ }^{\circ} \mathrm{C}$ overnight. Detection of alkaline phosphatase activity was performed using BCIP/NBT substrate (Roche Diagnostics $\mathrm{GmbH}$ ) at room temperature. Color development reaction was stopped in TRIS-NaCl buffer with EDTA $(1.212 \%$ TRIS, $0.876 \% \mathrm{NaCl}$, $0.0372 \%$ EDTA, $\mathrm{pH}=7.5$ ), sections were air-dried and coverslipped.

For $c$-fos and $v$ GluT2 double-labeling ISH, coronal sections were first hybridized with digoxigenin-labeled $c$-fos probes $(5 \mathrm{ng} / \mathrm{section}$ ) at $60{ }^{\circ} \mathrm{C}$ overnight. After washing with $2 \times$ SSC buffer, sections were hybridized with fluorescein-labeled $v$ GluT2 probes $(5 \mathrm{ng} / \mathrm{sec}-$ tion) at $60{ }^{\circ} \mathrm{C}$ overnight. The post-hybridization stringency washing steps were the same as described above for c-fos ISH. Sections were incubated with a mix of alkaline phosphatase-conjugated antidigoxigenin antibody (1:2500; Roche Diagnostics $\mathrm{GmbH})$ and antiFluorescein-POD antibody (1:1000; Roche Diagnostics $\mathrm{GmbH})$ at $4{ }^{\circ} \mathrm{C}$ overnight with alkaline phosphatase-conjugated anti-digoxigenin antibody against digoxigenin-labeled $c$-fos probes and antiFluorescein-POD antibody against fluorescein-labeled vGluT2 probes. Fluorescent signal was amplified using the Tyramide Signal Amplification (TSA) ${ }^{\mathrm{TM}}$ Kit ${ }^{*} 22$ (Ambion, Austin, TX, USA) by incubating the slides in fluorescein tyramide diluted amplification buffer (1:100) for $30 \mathrm{~min}$, followed by a further amplification step using VECTASTAIN ${ }^{\circ}$ ABC Kit (1:200) (Vector Laboratories, Burlingame, CA, USA). Finally, digoxigenin-labeled c-fos signal was detected using the filtered HNPP/Fast Red TR mix for $2 \times 20 \mathrm{~min}$ at room temperature. The incubation mix contained $10 \mu \mathrm{L}$ HNPP stock solution, $10 \mathrm{~mL}$ Fast Red TR stock solution and $1 \mathrm{~mL}$ detection buffer (HNPP/Fast Red TR system; Roche Diagnostics $\mathrm{GmbH}$ ).

\section{Image analysis}

Changes in IEG (or GAD65) expression may result in more cells being detected because the stimulus results in previously undetected cells synthesizing mRNA above detection levels. However, the existing (detected) population of cells might also produce more mRNA without significant changes in numbers. For this reason, both the number of $c$-fos- and GAD65-positive cells per surface area (i.e., cell density) and individual cellular labeling intensity [expressed as integrated optical density (OD)] were measured. A MCID Elite imaging system (Interfocus Imaging Ltd., Linton, UK) was used to quantify the numbers and/or individual labeling intensity of neurons immunopositive for $c$-fos, GAD65 or VGluT2 mRNA. Coronal sections of the habenula and RMTg were digitized using an objective magnification of $5 \times$ on a Leica DM/RBE photomicroscope connected to a digital camera (Evolution ${ }^{\mathrm{TM}}$ MP Color camera; Media Cybernetics, Rockville, MD, USA). The segmentation of $c$-fos and GAD65 positive cells from background was performed using an algorithm combining several point operators and spatial filters, aimed at detecting local changes in gray level and, thus, produce a measuring template for objects. Images went through histogram equalization, smoothing (low-pass filter, kernel size $7 \times 7$ ), and 
subtraction steps, and positive cells were detected using size and shape criteria (Gao et al., 2017).

In both the 10- and 60-days experiments, the cell density and labeling intensity of $c$-fos positive neurons were plotted against the rostrocaudal level of LHbL and LHbM (see Fig. 3). The mRNA levels of $c$-fos were examined at 10 levels from rostral to caudal of the LHbL (approximately from Bregma $-2.96 \mathrm{~mm}$ to Bregma $-4.1 \mathrm{~mm}$ ) and 13 levels from rostral to caudal of the LHbM (approximately from Bregma $-2.6 \mathrm{~mm}$ to Bregma $-4.1 \mathrm{~mm}$ ). The Bregma positions of each anatomical level are as follows: level $1=$ Bregma $-2.6 \mathrm{~mm}$, level $2=$ Bregma $-2.72 \mathrm{~mm}$, level $3=$ Bregma $-2.84 \mathrm{~mm}, \quad$ level $4=$ Bregma $-2.96 \mathrm{~mm}$, level $5=$ Bregma $-3.09 \mathrm{~mm}$, level $6=$ Bregma $-3.21 \mathrm{~mm}$, level $7=$ Bregma $-3.34 \mathrm{~mm}, \quad$ level $8=$ Bregma $-3.46 \mathrm{~mm}$, level $9=$ Bregma $-3.59 \mathrm{~mm}$, level $10=$ Bregma $-3.72 \mathrm{~mm}$, level $11=$ Bregma $-3.85 \mathrm{~mm}$, level $12=$ Bregma $-3.97 \mathrm{~mm}$, level $13=$ Bregma $-4.1 \mathrm{~mm}$. As the very rostral portion of the LHb consists of only the LHbM (Andres et al., 1999), the analysis of the LHbL comprises fewer anatomical levels than that of the LHbM. GAD65 mRNA levels were examined in the RMTg area and expressed as the average of three anatomical levels approximately at Bregma $-6.0 \mathrm{~mm}$ to Bregma $-6.5 \mathrm{~mm}$ (Bourdy \& Barrot, 2012). Parameters that were measured include the number of positive cells in each subregion, the subregional surface area and the integrated OD of labeled cell body (representing labeling intensity of positive cells). The number of positive cells per region of interest was expressed as cell density, that is, number of cells $/ \mathrm{mm}^{2}$.

For the analysis of $c$-fos and $v$ GluT2 mRNA co-expression, coronal section images (eight levels from Bregma $-3.09 \mathrm{~mm}$ to Bregma $-3.97 \mathrm{~mm}$ ) were captured using a Leica SP2 confocal laser scanning microscope (Leica Microsystems, Heidelberg, Germany). The scanning was performed with an objective magnification of $40 \times$, six focal planes and one airy unit of a spatial pinhole. The quality of digital images was improved by three times averaging, and the image size was expressed as $512 \times 512$ pixels. Double-labeled samples were sequentially scanned at wavelengths of 488 and $543 \mathrm{~nm}$. Fluorescence emission was then collected in the green and red regions of the spectrum. A MCID Elite imaging system (Interfocus Imaging Ltd.) was used for the imaging analysis of the maximum projected images to determine the co-localization of $c$-fos and $v G l u T 2$.

\section{Statistical analysis}

Data were analyzed using SPSS software 20 (IBM, New York, NY, USA). For the analysis of cocaine/sucrose self-administration behavior, a repeated measures ANOVA was performed with experimental group (i.e., cocaine self-administration, sucrose self-administration or control) as a between-subjects factor and self-administration session as a within-subject factor. A Greenhouse-Geisser correction was applied when Mauchly's test of sphericity indicated that the assumption of sphericity had been violated. To determine the effects of 10 or 60 days of cocaine/sucrose self-administration on c-fos expression, a repeated measures ANOVA was used with experimental group as a between-subjects factor and rostrocaudal level as a within-subject factor. Parameters measured included cell density (the number of $c$-fos-positive cells $/ \mathrm{mm}^{2}$ ) and labeling intensity (the integrated OD) of each individual cell body. Comparison of $c$-fos ISH after 10 and 60 days of cocaine self-administration was performed by normalizing the data from the cocaine groups to controls per rostrocaudal level and applying Student's $t$-test at each anatomical level. In addition, Student's $t$-test was used to test for differences in the percentages of cells in the LHbL co-expressing c-fos and vGluT2 after 10 and 60 days of cocaine self-administration. Finally, to determine the effects of cocaine self-administration on the GAD65 mRNA levels in RMTg, the cell density and the cellular labeling intensity of GAD65-positive neurons were analyzed by two-way ANOVA with experimental group and self-administration duration as between-subjects factors. Same as for c-fos, comparison of GAD65 ISH after 10 and 60 days of cocaine self-administration was performed by normalizing the data from the cocaine groups to controls.

\section{Results \\ Cocaine and sucrose self-administration behavior}

Rats were trained to self-administer cocaine or sucrose for either 10 or 60 days (Fig. 1). Responding on the active lever differed as a function of group and session in both the 10-day $\left[F_{\text {group }}(2,13)=25.07\right.$, $P<0.001 ; F_{\text {session }}(3.63,47.2)=1.74, P=0.162 ; F_{\text {group }} \times$ session $(7.27,47.2)=8.04, P<0.001)$ and 60 -day experiments $\left(F_{\text {group }}(2\right.$, $15)=181.9, P<0.001 ; F_{\text {session }}(6.97,104.5)=6.28, P<0.001$; $F_{\text {group }} \times$ session $\left.(13.94,104.5)=3.67, P<0.001\right]$ (Fig. 1). Post-hoc analysis showed that the number of rewards obtained in the sucrose group was significantly higher than that in the control and the cocaine groups in both experiments (both $P<0.001$ ). In the 60-day but not the 10-day experiment, the cocaine group obtained more rewards than the control group (10 days: $P=0.113$; 60 days: $P<0.001)$. When the numbers of active lever presses between the first and the final administration sessions were compared, significant increases were seen in the cocaine group (10 days: $t=-7.96, \mathrm{df}=10, P<0.001$; 60 days: $t=-10.30, \mathrm{df}=10, P<0.001)$, but not in the sucrose group (10 days: $t=-1.53$, df $=4.01, P=0.20 ; 60$ days: $t=-0.77$, df $=5, P=0.48$ ), whereas the number of active lever presses declined from the first to the final session in the control group in the 10-day $(t=3.54$, df $=8, P=0.008)$, but not in the 60-day experiment $(t=-1.13$, df $=10, P=0.282)$ (Fig. 1). The average number of active lever presses was significantly higher than the number of inactive responses in the cocaine (10 days: $t=6.02$, $\mathrm{df}=9.87$, $P<0.001 ; 60$ days: $t=34.72$, df $=93.7, P<0.001)$ and sucrose self-administration groups (10 days: $t=16.03$, df $=18, P<0.001$; 60 days: $t=60.62$, df $=75.33, P<0.001$ ) (data not shown). In the control group, no differences were seen between the number of active and inactive lever presses in either experiment (10 days: $t=-0.72$, df $=18, P=0.482 ; 60$ days: $t=1.82$, df $=118, P=0.071$ ) (data not shown).

\section{Cocaine self-administration induces c-fos expression in lateral habenula}

\section{Lateral part of lateral habenula}

Animals were killed and the tissue was collected for $c$-fos visualization $30 \mathrm{~min}$ after the final training session. The structure of LHb varies from rostral to caudal, with LHbM present through the whole structure while LHbL occurring more caudally (Fig. 2). The distribution of c-fos ISH signal in LHb showed a heterogeneous pattern through the rostral-caudal extent, but also differed between the medial and lateral parts in specific portions (Fig. 2). In the sucrose and control groups, the $c$-fos signal in the LHb was generally very low. By contrast, in the cocaine self-administration group, we observed two groups of cells in the LHb. In most cases, the group with stronger signals was located in the LHbL (Fig. 2B and C). The density of labeled neurons in the LHbL increased from the rostral to the mid-rostrocaudal levels of the structure (approximately from Bregma 

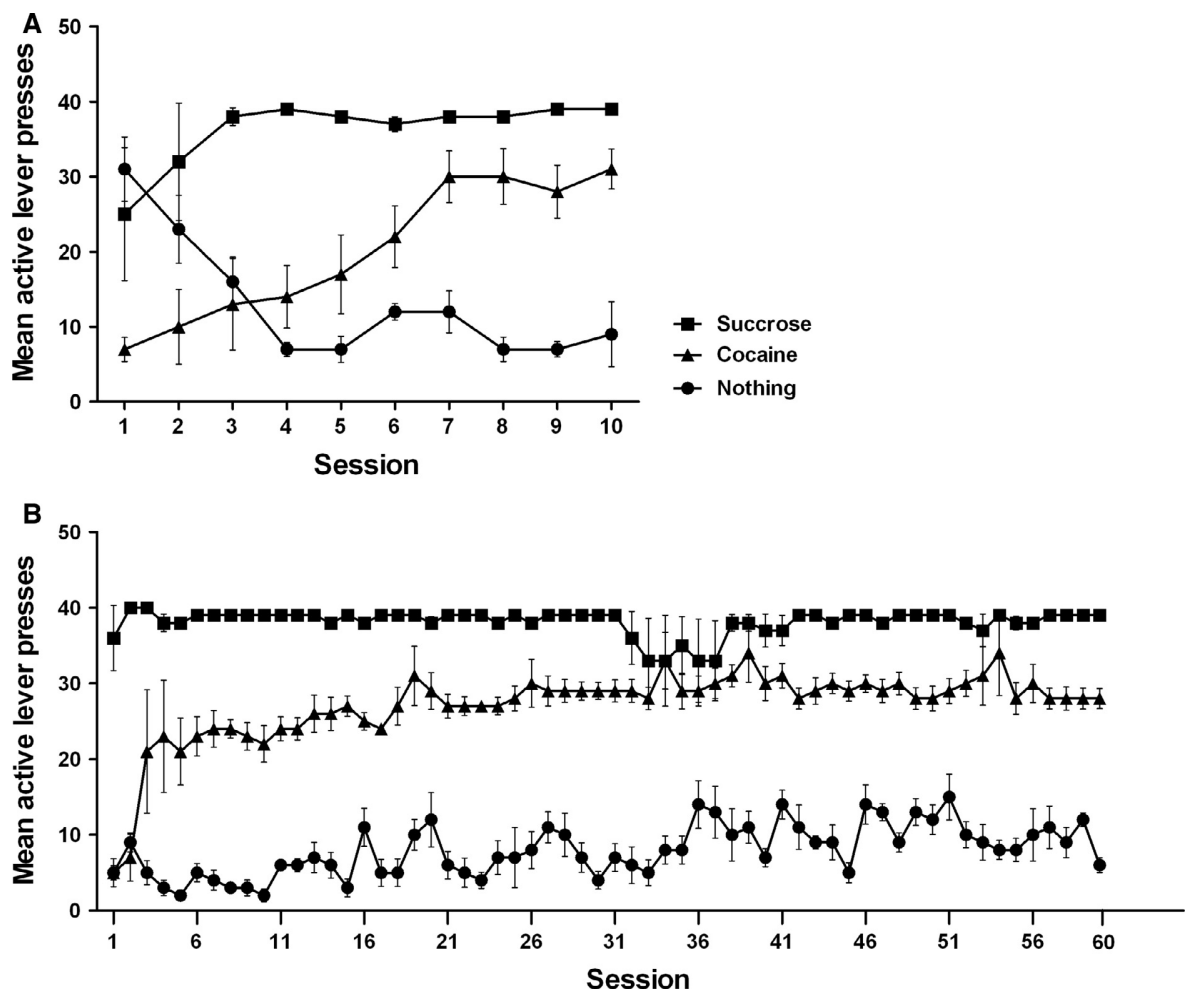

FIG. 1. Short-term (10 days, A) vs. long-term (60 days, B) self-administration of cocaine or sucrose. Data are presented as mean \pm SEM number of active lever presses over animals per group.

$-3.21 \mathrm{~mm}$ to Bregma $-3.97 \mathrm{~mm}$ ) and then decreased again to low levels in the most caudal part of the LHbL after cocaine self-administration (Fig. 3). This was observed in both the $10-\left[F_{\text {group }}(2,12)\right.$ $=81.95, \quad P<0.001 ; \quad F_{\text {level }}(2.27, \quad 27.20)=3.14, \quad P=0.054$; $\left.F_{\text {group } \times \text { level }}(4.53,27.20)=4.15, P=0.008\right]($ Figs 2 and $3 \mathrm{~A})$ and the 60-day experiments $\left[F_{\text {group }}(2,14)=22.38, P<0.001 ; F_{\text {level }}\right.$ $(2.14,30.02)=3.94, P=0.028 ; F_{\text {group }} \times$ level $(4.29,30.02)=3.75$, $P=0.012$ ] (Fig. 3B). Post-hoc tests revealed that the density of $c$-fos-labeled cells was significantly enhanced in the cocaine group compared with the control and sucrose groups in both the 10 days $(P<0.001)$ and 60 days experiments $(P<0.001)$. No differences were found between the control and sucrose groups. In the 10-day experiment, differences between cocaine and the other two groups were found at rostrocaudal levels 6-12 (approximately from Bregma $-3.21 \mathrm{~mm}$ to Bregma $-3.97 \mathrm{~mm}$ ) in the LHbL (cocaine vs. control: level 6, $P=0.006$; level 7, $P=0.009$; level 8, $P<0.001$; level 9, $P<0.001$; level 10, $P<0.001$; level 11, $P=0.033$; level 12, $P=0.005$; cocaine vs. sucrose: level $6, P=0.014$; level 7 , $P=0.004$; level $8, P<0.001$; level 9, $P<0.001$; level 10 , $P<0.001$; level $11, P=0.04$; level $12, P=0.006$ ). In the 60 -day experiment, the cocaine group differed from the control and sucrose groups at levels 7-12 (approximately from Bregma $-3.34 \mathrm{~mm}$ to Bregma $-3.97 \mathrm{~mm}$ ) (cocaine vs. control: level 7, $P=0.012$; level $8, P<0.001$; level $9, P<0.001$; level $10, P=0.028$; level 11 , $P=0.005$; level 12, $P<0.001$; cocaine vs. sucrose: level 7 , $P=0.009$; level $8, P<0.001$; level $9, P<0.001$; level 10 , $P=0.028$; level 11, $P=0.005$; level 12, $P<0.001$ ) (Fig. 3A and B). Taken together, both 10 and 60 days of cocaine self-administration evoked an increased density of $c$-fos-positive neurons in the $\mathrm{LHbL}$ that displayed a rostrocaudal gradient with a peak in cell numbers at level 8 (Bregma $-3.46 \mathrm{~mm}$ ).

As the density of $c$-fos-positive cells was increased at levels 7-12 in the LHbL after both short- and long-term cocaine self-administration, we further analyzed whether the cocaine-induced increases in density of $c$-fos-positive neurons differed between the 10- and 60-day experiments at these levels. Student's $t$-test showed that at two levels, cocaine exposure-induced increases in the density of $c$-fos neurons were significantly higher after 10 days than after 60 days (level 8: $t=3.05$, $\mathrm{df}=10, P=0.012$; level 10: $t=3.42$, $\mathrm{df}=10, P=0.007$ ) (Fig. 3E). No differences were seen between the experiments at the other four levels (level 7: $t=0.27$, df $=10$, $P=0.799$; level 9: $t=0.07, \mathrm{df}=10, P=0.945$; level 11: $t=0.72$, $\mathrm{df}=10, \quad P=0.489 ; \quad$ level $12: \quad t=0.58, \quad \mathrm{df}=10, \quad P=0.574)$ (Fig. 3E).

For the cellular labeling intensity of $c$-fos-positive cells in the LHbL, there was a main effect of group and anatomical level $\left[F_{\text {group }}(2,12)=8.11, \quad P=0.006 ; \quad F_{\text {level }}(3.91,46.94)=2.95\right.$, $P=0.031]$ after 10 days of self-administration. However, the interaction between group and anatomical level was not significant $\left[F_{\text {group }} \times\right.$ level $\left.(7.82,46.94)=1.48, P=0.193\right]$ (Fig. 3C). Post-hoc testing showed that the density of $c$-fos-positive cells in the cocaine group was significantly higher than that in the control $(P=0.044)$ and sucrose groups $(P=0.006)$. When animals were exposed to cocaine or sucrose for 60 days, neither the group nor the anatomical level had a significant main effect on the intensity of $c$-fos-positive cells $\left[F_{\text {group }}(2,14)=1.77, P=0.207 ; F_{\text {level }}(3.58,50.08)=1.14\right.$, $P=0.347 ; F_{\text {group }} \times$ level $\left.(7.15,50.08)=0.74, P=0.639\right]($ Fig. 3D) . To summarize, 10 days of cocaine self-administration increased the labeling intensity of $c$-fos-positive cells in the LHbL, but no changes were observed after 60 days of cocaine self-administration.

\section{Medial part of lateral habenula}

After 10 days of self-administration, there were no differences in the density of $c$-fos-labeled neurons between the experimental groups, although $c$-fos cell density differed as a function of 

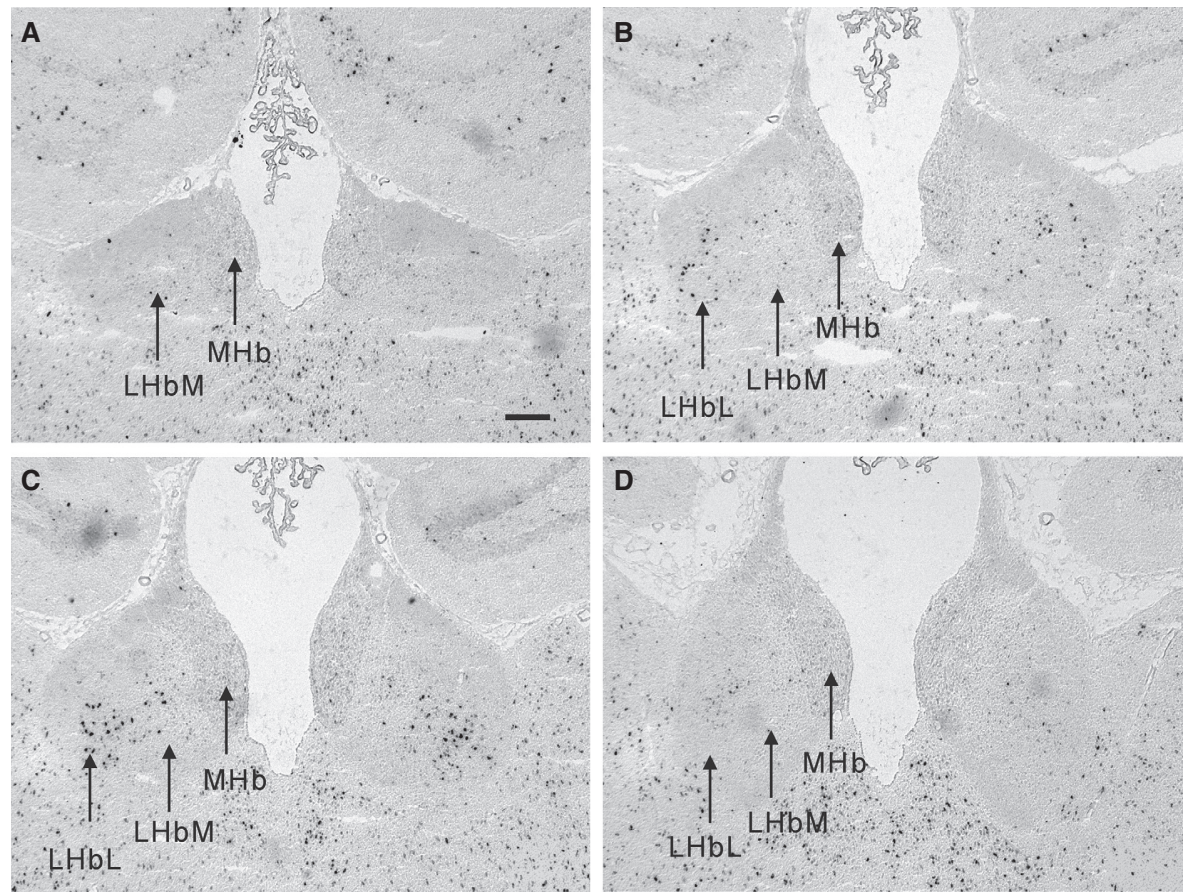

FIG. 2. Representative micrographs of $c$-fos hybridized sections in habenula. Two subregions including medial habenula (MHb) and medial part of lateral habenula $(\mathrm{LHbM})$ are present throughout the rostral-caudal axis of habenula (A-D), whereas the lateral part of lateral habenula (LHbL) is present more caudally (B-D). Ten days of cocaine self-administration had no effects on $c$-fos expression in the most rostral and caudal level of lateral habenula (LHb) (A and D; A, Bregma $-2.72 \mathrm{~mm}$, level 2; D, Bregma $-4.1 \mathrm{~mm}$, level 13), but increased $c$-fos in situ hybridization (ISH) signal in the mid-rostrocaudal portion (B and C; B, Bregma $-3.21 \mathrm{~mm}$, level 6; C, Bregma $-3.59 \mathrm{~mm}$, level 9). Scale bar: $200 \mu \mathrm{m}$ in A-D.

anatomical level $\left[F_{\text {group }}(2,11)=2.89, P=0.098 ; F_{\text {level }}(3.90\right.$, $42.93)=3.82, \quad P=0.01 ; \quad F_{\text {group }} \times$ level $\quad(7.80, \quad 42.93)=1.79$, $P=0.107$ ] (Figs 2 and 4A). After 60 days of cocaine self-administration, there was an increase in the density of $c$-fos-positive cells in the $\mathrm{LHbM}$ as a function of anatomical level $\left[F_{\text {group }}(2,15)=11.06\right.$, $P=0.001 ; \quad F_{\text {level }}(5.34, \quad 80.06)=3.18, \quad P=0.01 ; \quad F_{\text {group } \times \text { level }}$ $(10.67,80.06)=3.25, P=0.001]$ (Fig. 4B). Post-hoc tests revealed that 60 days of cocaine exposure significantly enhanced the density of $c$-fos-positive cells in the LHbM compared to the control $(P=0.001)$ and the sucrose $(P=0.002)$ groups (Fig. 4B). The differences between cocaine and the other two groups were seen at rostrocaudal levels 7-9 (cocaine vs. control: level 7, Bregma -3.34 , $P=0.003$; level 8, Bregma $-3.46, P<0.001$; level 9, Bregma $-3.59, P=0.002$; cocaine vs. sucrose: level $7, P=0.048$; level 8 , $P<0.001$; level 9, $P=0.005$ ) (Fig. 4B). Since the 60 days of cocaine self-administration significantly increased the density of $c$ fos-positive cells at three levels (7-9) in LHbM, we further examined whether this effect was different from that after 10 days at these levels. Student's $t$-test showed no significant differences between 10 and 60 days of cocaine self-administration (level 7: $t=-2.01, \mathrm{df}=10, P=0.073$; level 8: $t=0.31$, df $=10, P=0.77$; level 9: $t=-1.94$, $\mathrm{df}=6.36, P=0.098$ ). In summary, 60 days, but not 10 days of cocaine self-administration enhanced the density of c-fos-positive cells in the LHbM.

Cocaine or sucrose self-administration did not affect the labeling intensity of $c$-fos-positive neurons in the LHbM, although there were minor rostrocaudal differences [10 days: $F_{\text {group }}(2,11)=2.53$, $P=0.124 ; F_{\text {level }}(5.38,59.22)=2.59, P=0.031 ; \quad F_{\text {group } \times \text { level }}$ $(10.77,59.22)=1.43, P=0.185 ; 60$ days: $F_{\text {group }}(2,15)=0.73$, $P=0.50 ; \quad F_{\text {level }}(3.77, \quad 56.52)=1.88, \quad P=0.130 ; \quad F_{\text {group }} \times$ level $(7.54,56.52)=0.85, P=0.561]$ (Fig. $4 \mathrm{C}$ and D).

\section{The majority of c-fos-positive neurons in the $\mathrm{LHbL}$ is glutamatergic}

To test whether the $c$-fos-positive cells that were induced by the cocaine self-administration were glutamatergic, we performed a double ISH for $c$-fos and $v G l u T 2$. As shown in Fig. 5A-C, most of the c-fos-positive cells in the LHbL expressed vGluT2 mRNA. After 10 days cocaine exposure, $75.5 \pm 1.7 \% \quad c$-fos-labeled cells were $v$ GluT2 positive, and $75.7 \pm 2.4 \%$ were $v$ GluT2 positive after 60 days of cocaine self-administration (Fig. 5D). There were no differences in the percentage of $c$-fos-labeled cells that expressed $v$ GluT2 between the 10- and 60-days cocaine groups $(t=-0.051$, df $=4, P=0.962$ ). The identity of the vGluT2-negative $c$-fos-labeled cells was not established. The latter cells might have a different phenotype; however, they might also produce low levels of VGlut2 that -in our hands - remained under the detection limit of the double-labeling procedure. Evidence in the literature suggests that a GABAergic phenotype is not very likely (Brinschwitz et al., 2010; Li et al., 2011; Aizawa et al., 2012).

\section{Increases of GAD mRNA in RMTg after 10 days but not 60 days cocaine self-administration}

The density and labeling intensity of GAD65-positive cells in RMTg were compared between the cocaine-exposed groups and control. The sucrose groups were omitted because sucrose self-administration did not affect $c$-fos expression in the habenula. Ten days of cocaine selfadministration increased the GAD65 ISH signals in RMTg (Fig. 6B) when compared to the control group (Fig. 6A). In contrast, after 60 days of cocaine self-administration, no obvious differences were observed between the control and cocaine groups (Fig. 6C and D). 

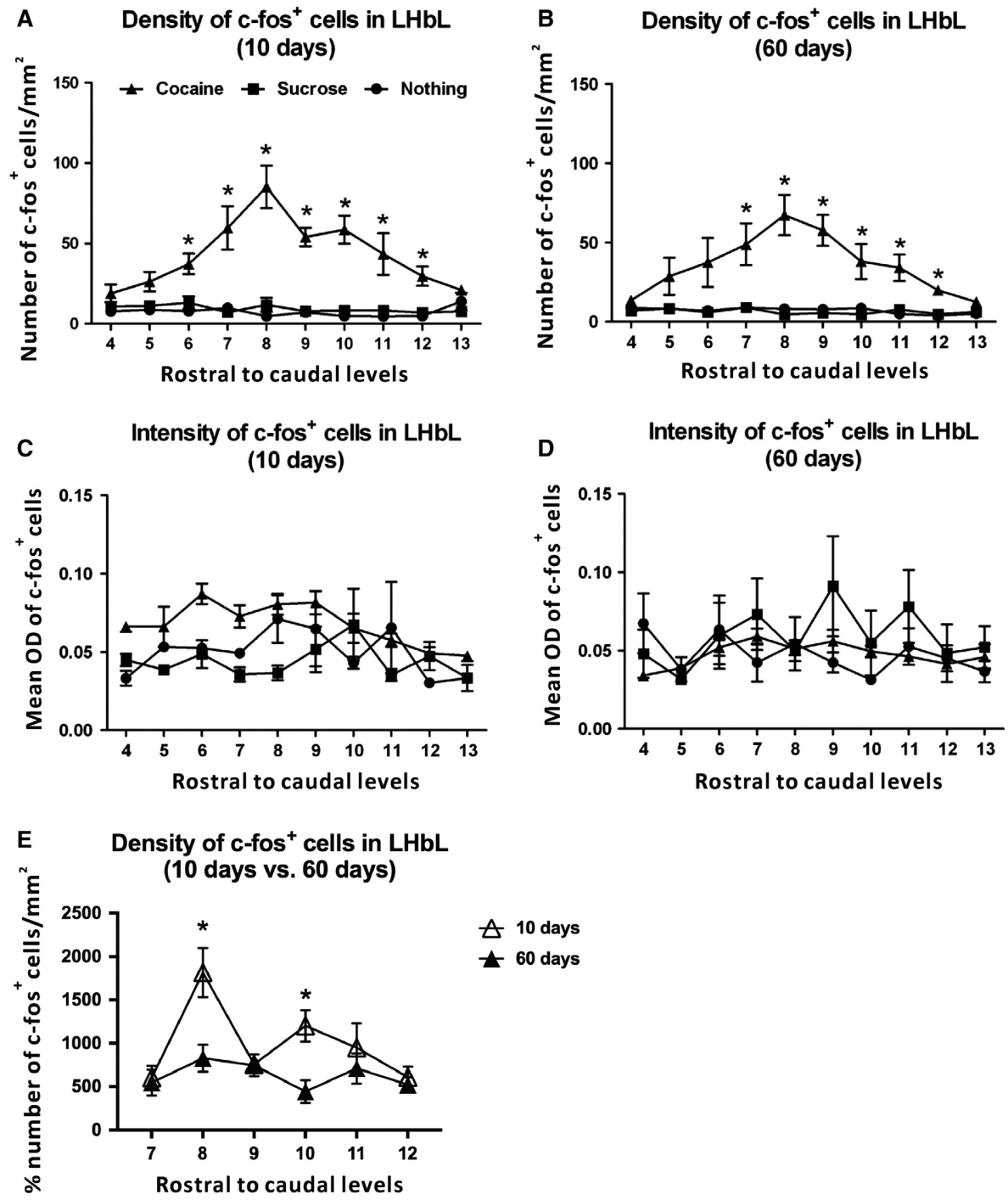

FIG. 3. Changes in density and labeling intensity of c-fos-positive cells along the rostrocaudal axis in lateral part of lateral habenula (LHbL) after cocaine or sucrose self-administration. Left panels $(\mathrm{A}, \mathrm{C})$ show results after 10 days self-administration, right panels (B, D) after 60 days self-administration. $X$-axis represents 10 anatomical levels from rostral to caudal in A-D. Differences in the density of $c$-fos-positive cells between 10 and 60 days cocaine groups were compared at six anatomical levels $(\mathrm{E})$. Values are presented as mean \pm SEM cell density or optical density. ${ }^{*} P<0.05$, significant difference between cocaine and control/sucrose groups.

Two-way ANOva demonstrated that the cocaine-induced increases in the density of $G A D 65$-labeled neurons were affected by the duration of self-administration $\left[F_{\text {group }}(1,16)=3.93, P=0.065 ; F_{\text {duration }}(1,16)\right.$ $=11.02, P=0.004 ; F_{\text {group }} \times$ duration $\left.(1,16)=11.04, P=0.004\right]$. Post-hoc tests revealed that, compared to control, the density of GAD65-positive neurons increased significantly in the cocaine group in the 10-day experiment $(t=-4.19$, df $=7.40, P=0.004)$, but not in the 60-day experiment $(t=-0.98, \mathrm{df}=8, P=0.354)$. In addition, the density of $G A D 65$-positive neurons in the 10 days cocaine group was higher than that in the 60-days cocaine group $(t=4.53$, df $=10, P=0.001$ ) (Fig. 6E).

The labeling intensity of the GAD65-positive cells in the RMTg was also affected by cocaine self-administration in a manner that depended upon self-administration experience $\left[F_{\text {group }}(1,16)\right.$ $=19.06, \quad P<0.001 ; \quad F_{\text {duration }}(1, \quad 16)=14.25, \quad P=0.002$; $F_{\text {group }} \times$ duration $\left.(1,16)=14.3, P=0.002\right]$. Post-hoc tests revealed that after 10 days of self-administration, the intensity of GAD65labeled neurons was significantly increased in the cocaine group
( $t=5.14, \mathrm{df}=8, P=0.001)$. After 60 days of self-administration, there was no difference between the cocaine self-administration and the control groups $(t=0.49$, df $=8, P=0.644)$. In addition, the intensity of GAD65-positive neurons in the 10 days cocaine group was significantly higher than in the 60 days cocaine self-administration group ( $t=6.05, \mathrm{df}=10, \quad P<0.001) \quad$ (Fig. 6F). In summary, 10 days of cocaine self-administration significantly increased both the density and the labeling intensity of GAD65-positive neurons in RMTg, while after 60 days no differences were seen between the cocaine and the control groups.

\section{Discussion}

The aim of the present study was to examine the consequences of prolonged drug taking on neuronal activity in subregions of the $\mathrm{LHb}$. Increased expression of $c$-fos was primarily found in the lateral part of the LHb after both short- and long-term self-administration of cocaine. The cocaine-exposed animals not only displayed a higher 

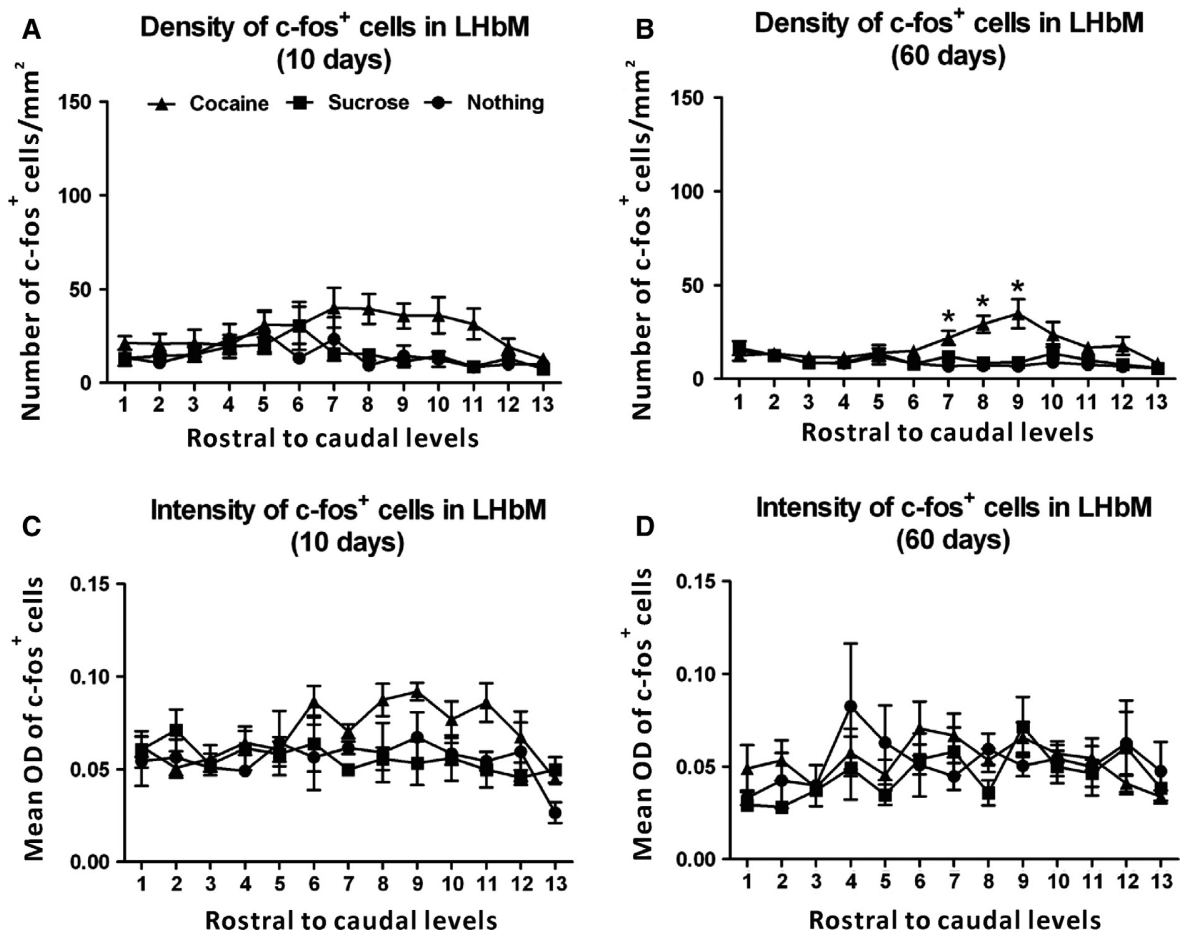

FIG. 4. Changes in density and labeling intensity of $c$-fos-positive cells along the rostrocaudal axis in medial part of lateral habenula (LHbM) after cocaine or sucrose self-administration. Left panels (A, C) show results after 10 days self-administration, right panels (B, D) after 60 days self-administration. $X$-axis represents 13 anatomical levels from rostral to caudal. Values are presented as mean \pm SEM cell density or optical density. ${ }^{*} P<0.05$, significant difference between cocaine and control/sucrose groups.
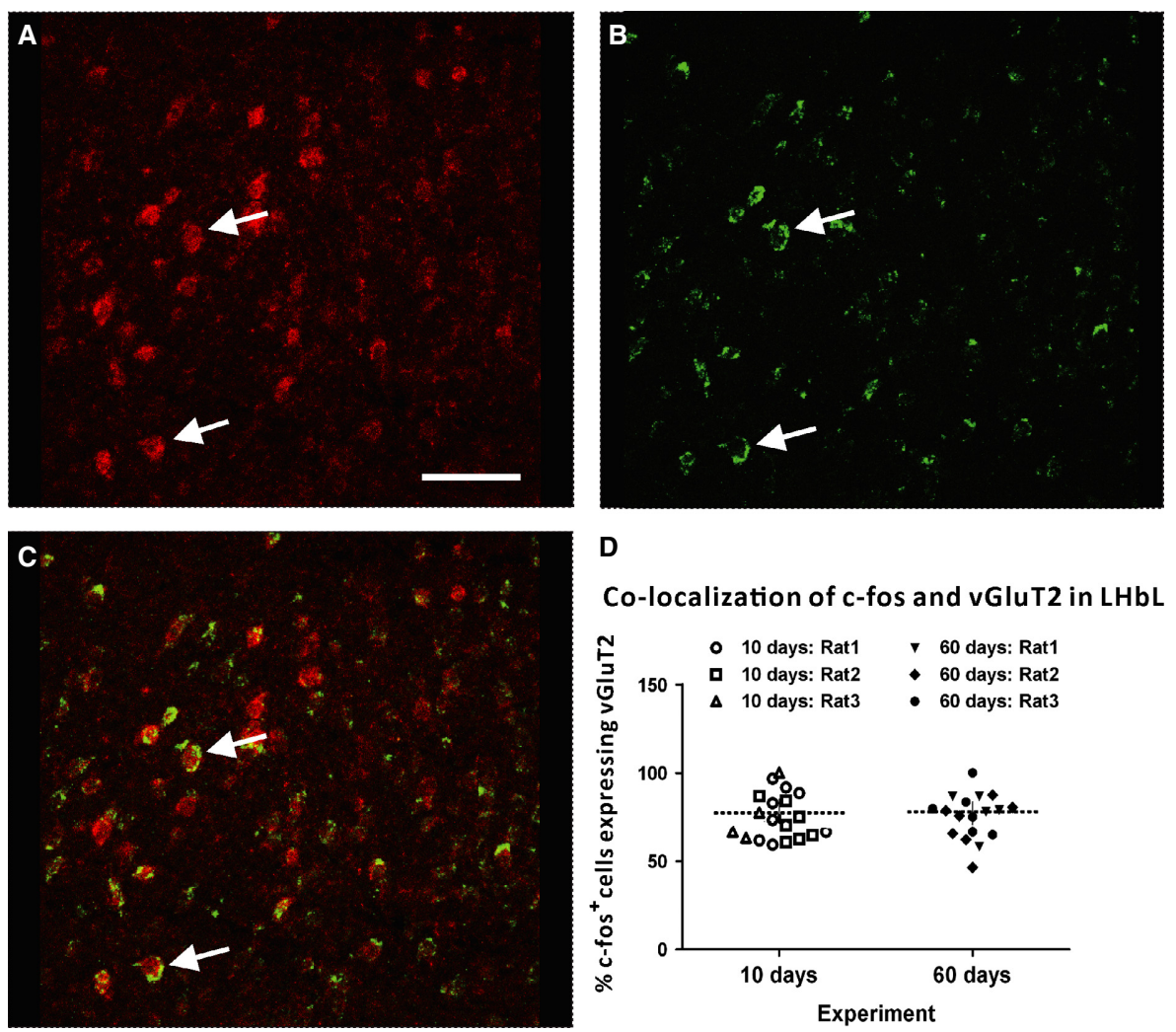

FIG. 5. Double-labeling in situ hybridization of $c$-fos mRNA (red in A) and $v G l u T 2$ mRNA (green in B) and the merged images (C) in the lateral part of lateral habenula $(\mathrm{LHbL})$ after 10 days of cocaine self-administration. 'Arrows' point to examples of cells that are both $c$-fos and $v G l u T 2$ positive. (D) Percentages of c-fos-positive cells expressing $v$ GluT2 in LHbL after 10 and 60 days of cocaine self-administration. Scale bar: $75 \mu \mathrm{m}$ in A-C. [Colour figure can be viewed at wileyonlinelibrary.com]. 

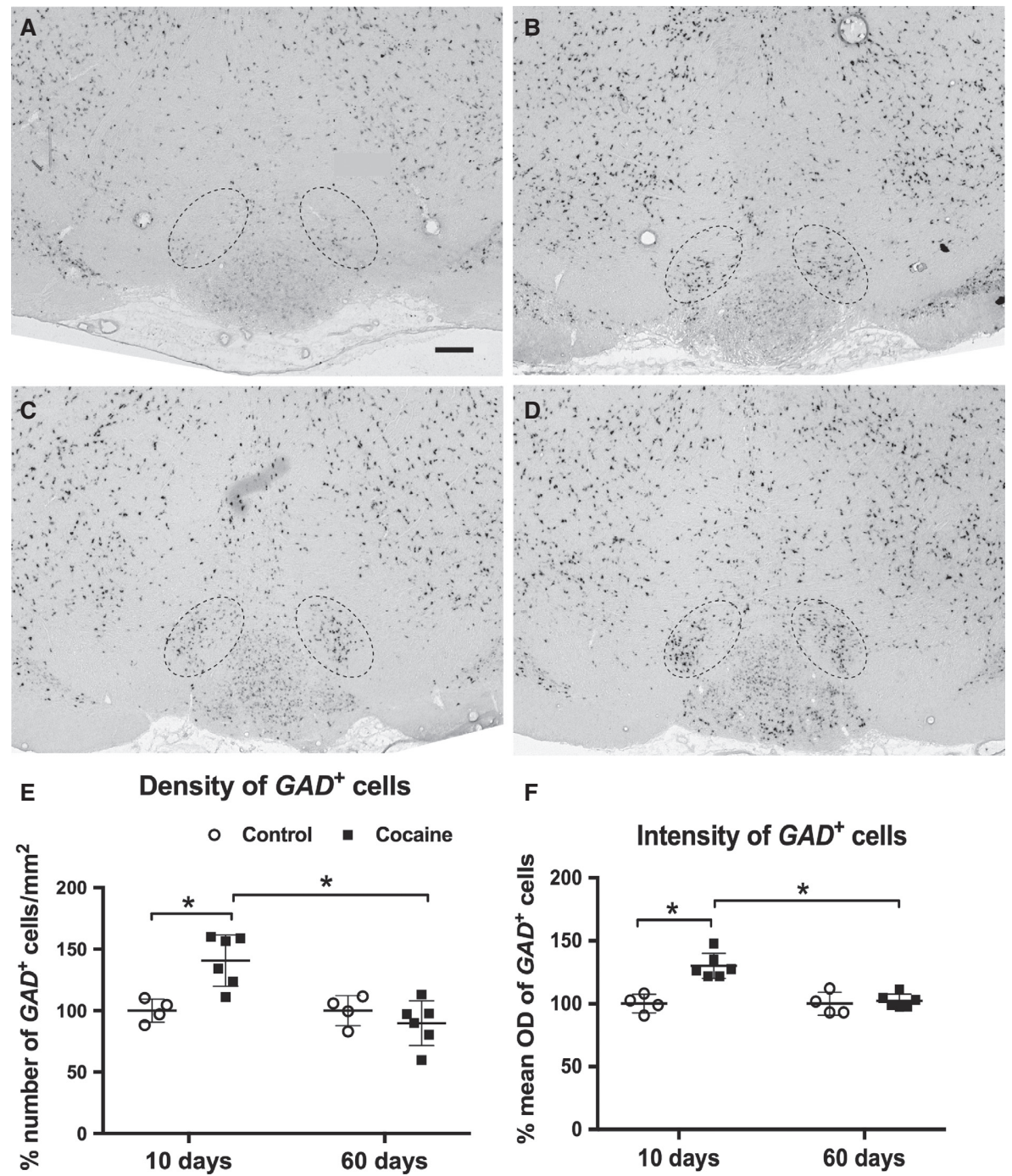

FIG. 6. Changes in GAD-65 expression in rostral mesencephalic tegmental nucleus (RMTg) after short- or long-term cocaine self-administration. Four representative micrographs of hybridized coronal sections show immunostaining of GAD-65 probe after 10 days of cocaine self-administration in the control (A) and cocaine (B) groups and after 60 days in controls (C) and cocaine-exposed animals (D). The density (E) and labeling intensity (F) of GAD-65-positive cells in RMTg are expressed as mean \pm SEM percentage of control. ${ }^{*} P<0.05$, significant difference between groups. Scale bar: $200 \mu$ m in A-D.

number of $c$-fos-positive neurons in the LHbL but also enhanced response intensity of the individual reactive cells. The increase in reactive cell numbers was similar after short- and long-term cocaine self-administration. However, the augmented cellular response intensity was no longer significant after 60 days of cocaine taking. Cocaine self-administration did not alter cellular reactivity in the LHbM after short-term drug taking, but after 60 days of self-administration, an increase in the number of $c$-fos-positive cells was found in the LHbM. Interestingly, the distribution of $c$-fos-positive cells was far from homogeneous as most reactive neurons were located in the mid-rostrocaudal part of habenula. The majority of activated neurons in the LHbL was vGluT2-positive, that is, glutamatergic, after both 10 and 60 days of cocaine self-administration. Furthermore, in the RMTg, which is a principal output structure of the $\mathrm{LHb}$, both the number of GABAergic cells and their individual response intensity were upregulated after short-term but not after long-term cocaine selfadministration. Together, these data reveal the complex temporal dynamics by which cocaine self-administration alters activity in
LHb circuitry, which may play an important role in the underlying mechanisms of drug taking with prolonged cocaine use experience.

\section{Heterogeneous neuronal activation in $\mathrm{LHb}$}

c-fos neurons in the present report displayed a highly heterogeneous distribution that was first and foremost characterized by a gradient with a peak in the numbers of $c$-fos-positive neurons in the $\mathrm{LHbL}$ and LHbM centering at (approximately) Bregma levels $-3.32 \mathrm{~mm}$ to $-3.72 \mathrm{~mm}$. This response pattern may be related to the subnuclear organization of the LHb, although a clear distinction between subnuclei cannot be made on basis of rostrocaudal location (Andres et al., 1999; Geisler et al., 2003). Besides size and shape of the habenular subnuclei, differences that were found along the rostrocaudal extent of LHb in (immuno-) histochemical characteristics indicative of differences in cellular phenotype may be related to the present heterogeneous functional response to cocaine selfadministration (Geisler et al., 2003). 
Comparison of the rostrocaudal response pattern of $c$-fos expression after cocaine self-administration with structural connectivity of LHb does not readily reveal a resemblance. Whereas Goncalves et al. (2012) found projections from LHb to VTA to originate mainly from medial and caudal regions, Petzel et al. (2017) in the rat and Quina et al. (2015) in the mouse demonstrated projections to VTA and raphe nuclei that are widely distributed throughout the LHb's rostrocaudal extent. With respect to dopaminergic input, the dopamine transporter - a primary target of cocaine - has been shown to be primarily expressed in the parvocellular and central subnuclei of the medial division of the LHb complex which are found along the rostrocaudal length of LHb (Geisler et al., 2003).

In a number of previous studies, a differentiation in the activation of the lateral and medial parts of LHb has been described. For example, following the systemic administration of dopamine receptor agonists, neurons were activated only in the LHbL (Wirtshafter et al., 1994; Kowski et al., 2009). Interestingly, short-term (i.e., one of six sessions) cocaine self-administration has been found to increase $c$-fos expression in both LHbL and in LHbM (Zahm et al., 2010). In the latter study, the response in the LHbL was comparable between one and six sessions, whereas the response in the LHbM declined between the first and sixth cocaine self-administration session. In the present study, we observed two clusters of $c$-fos-labeled cells in the LHb after cocaine self-administration, with the larger one situated in the LHbL and the smaller one in the LHbM (see Fig. 2). The activational response was much stronger in LHbL than LHbM. Comparison with the subnuclear organization of $\mathrm{LHb}$ as described by Andres et al. (1999) suggests that the larger cluster of $c$-fos-positive cells in the LHbL in our study is situated in the oval part of the LHbL (LHbLO); the smaller cluster of activated neurons in the LHbM is presumably located in the parvocellular and/or the central part of the LHbM (LHbMPc and/or LHbMC) (Andres et al., 1999). Unfortunately, due to technical restrictions, we were not able to relate the location of the activated neurons precisely to the fine subnuclear division (Andres et al., 1999; Geisler et al., 2003).

The more global medial-lateral division of the LHb as demonstrated in our study appears relevant in the context of organization of the afferent and efferent connections of the habenula (Lecourtier \& Kelly, 2007). Neuroanatomical tracing studies have shown that the LHbM predominantly receives afferents from limbic-related areas such as the lateral preoptic and lateral hypothalamic areas, whereas the LHbL collects primarily inputs from the entopeduncular nucleus (Herkenham \& Nauta, 1977; Lecourtier \& Kelly, 2007; Hikosaka et al., 2008). Thus, the strongest response to cocaine selfadministration in the present paradigm occurred in the basal gangliarelated portion of the LHb. As regards its efferents, the LHb is known to issue fibers to VTA, the substantia nigra complex and adjacent tegmental nuclei, as well as to the raphe nuclei and the lateral hypothalamus (Herkenham \& Nauta, 1979; Barrot et al., 2012). Direct projections from $\mathrm{LHb}$ to the dopaminergic VTA originate predominantly in the LHbM, as do efferents to the 5HT system (Goncalves et al., 2012; Proulx et al., 2014; Sego et al., 2014). However, with respect to efferent control over dopaminergic cell groups, LHb projections - arising from its lateral part - primarily target the rostral midbrain tegmental nucleus (RMTg), which forms a GABAergic intermediate between the habenula and the dopaminergic neurons in the VTA (Jhou et al., 2009b; Kaufling et al., 2009; Omelchenko et al., 2009; Brinschwitz et al., 2010; Balcita-Pedicino et al., 2011; Proulx et al., 2014). The enhanced $c$-fos expression in the present experiments that was observed in LHbL and LHbM therefore signifies a stronger influence on different aspects of reward processing involving dopaminergic and serotoninergic mechanisms after cocaine self-administration (see Proulx et al., 2014; for review). Our finding that upregulation of $c$-fos was more robust in the LHbL suggests an important effect on dopaminergic neurotransmission. That is, increased output of the LHbL may reduce firing activity of dopaminergic neurons via its glutamatergic projections to the GABAergic neurons in RMTg. Indeed, a glutamatergic identity could be established for the majority of $c$-fos neurons in the present experiments, as could an augmentative effect on GAD65 expression in RMTg. The latter findings are in line with the cocaine-induced c-fos activation that has been reported in the LHbL-to-RMTg projections (Jhou et al., 2013).

\section{Neuronal activity in $\mathrm{LHb}$ after short- and long-term cocaine use}

Cocaine has been shown to both decrease and increase the firing rate of LHb neurons, with the inhibitory effect preceding excitation (see Lecca et al., 2014 for review). The increase in firing frequency may be prolonged and this response is likely related to the $c$-fos activation seen in the present study and in other work (Jhou et al., 2013). Previous reports have shown that exposure to cocaine, through stimulation of dopamine receptors, increases glutamate function in the LHb (Maroteaux \& Mameli, 2012; Jhou et al., 2013; Zuo et al., 2013; Neumann et al., 2014; Meye et al., 2015), which then leads to changes in post-synaptic AMPA receptor function (Maroteaux \& Mameli, 2012; Meye et al., 2015). This provides a likely underlying mechanism for the increases in $c$-fos expression observed in the present study.

As LHb neurons are excited by aversive states and inhibited by unexpected rewarding events, their activation thus results in inhibition of dopaminergic neurons and suppression of appetitive behavior (Matsumoto \& Hikosaka, 2009; Bromberg-Martin et al., 2010a; Bromberg-Martin \& Hikosaka, 2011). Interestingly, also activation of the direct pathway from LHb to VTA appears to have aversive effects (Lammel et al., 2012). This means that the over-activity in $\mathrm{LHb}$ after cocaine self-administration that was observed in the present study likely represents increased inhibition of dopaminergic neurons, a process that may be associated with aversive effects evoked by cocaine. Dopaminergic and glutamatergic mechanisms in the habenula-besides playing a likely role in the observed increase in $c$-fos expression-have been shown to be involved in the aversive effects of cocaine exposure (Jhou et al., 2013; Meye et al., 2015; Shelton et al., 2016). Moreover, it has been demonstrated that intact $\mathrm{LHb}$ function is necessary for rats to inhibit responding to cocaineassociated cues when it is signaled that cocaine is not available (Mahler \& Aston-Jones, 2012; Zapata et al., 2017), although there is no evidence that the habenula is involved in punished cocaine seeking (see Jean-Richard-Dit-Bressel \& McNally, 2014; Zapata et al., 2017). Thus, the LHb may subserve the ability to inhibit drug use on the basis of internal (aversive- or expected aversive effects of the drug) or external stimuli (indicating unavailability of cocaine).

Importantly, prolonged cocaine exposure has been shown to result in impaired function of the LHb and its outputs (Meshul et al., 1998; see also Zahm et al., 2010; Lax et al., 2013). In the context of these data, our findings suggest that the influence of the LHb over cocaine use changes with prolonged drug-taking experience. In early stages of drug use (i.e., after 10 days of self-administration), the lateral portion of the LHb, perhaps by its output to the RMTg, may serve to control drug intake by encoding its negative aspects. After extensive cocaine-taking experience, however, the $\mathrm{LHb}$ involvement shifts somewhat to its medial portion and the 
LHbL-RMTg projection becomes disengaged, as evidenced by the fact that increased $G A D$ expression in the RMTg was found after 10 days of self-administration only. As a result, the influence of aversive aspects of drug use on behavior may decline, which provides a neural pathway by which cocaine taking becomes less sensitive to adversity (Deroche-Gamonet et al., 2004; Vanderschuren \& Everitt, 2004; Vanderschuren \& Ahmed, 2013; for reviews see Vanderschuren et al., 2017). The latter is a hallmark of addictive behavior. Future behavioral studies should directly investigate this possibility and, in the process, should establish the hypothesized causal relationship between LHbL activation and GABAergic activity in RMTg.

In conclusion, the present study demonstrates a strongly heterogeneous neuronal activation pattern in LHb after cocaine self-administration. After short-term cocaine exposure, increased neuronal activity predominated in the basal ganglia-related LHbL, whereas after long-term cocaine self-administration, the limbic-related LHbM became more engaged. We speculate that this process leads to increased $\mathrm{LHb}$ output to RMTg after short-term drug exposure resulting in enhanced inhibitory control over mesencephalic dopaminergic cells and that this effect abates after long-term drug use. Our finding of increased metabolic activity in the RMTg after 10 days of self-administration but not after 60 days supports this conjecture. As the LHb is implicated in the processing of negative emotional stimuli, signaling aversive states related to cocaine taking may be attenuated after prolonged drug use, possibly leading to diminished reluctance to engage in drug self-administration.

\section{Acknowledgements}

Supported by ZonMw (the Netherlands Organisation for Health Research and Development) Grant 91207006 (awarded to L.J.M.J. Vanderschuren., P. Voorn and A.B. Smit). We thank Jules Limpens for carrying out the behavioral experiments, and Allert Jan Jonker and Evelien Timmermans-Huisman for their help with the histological experiments

\section{Conflict of interest}

All authors declare no conflict of interest.

\section{Author contributions}

PV, HJG and LJMJV contributed to the study concept and design. PG performed the experiments and data analysis. PG and HJG drafted the manuscript. PV and LJMJV provided critical revision of the manuscript. All authors critically reviewed content and approved final version for publication.

\section{Data accessibility}

Data are available from the corresponding author on request.

\section{Abbreviations}

IEG, immediate early gene; ISH, in situ hybridization; LHb, lateral habenula; LHbL, lateral part of lateral habenula; LHbM, medial part of lateral habenula; RMTg, rostral mesencephalic tegmental nucleus; VTA, ventral tegmental area.

\section{References}

Aizawa, H., Kobayashi, M., Tanaka, S., Fukai, T. \& Okamoto, H. (2012) Molecular characterization of the subnuclei in rat habenula. J. Comp. Neurol., 520, 4051-4066.
American Psychiatric Association (2013) Diagnostic and Statistical Manual of Mental Disorders. Fifth Edition (DSM-5). American Psychiatric Association, Washington, DC, USA.

Andres, K.H., von During, M. \& Veh, R.W. (1999) Subnuclear organization of the rat habenular complexes. J. Comp. Neurol., 407, 130-150.

Araki, M., McGeer, P.L. \& Kimura, H. (1988) The efferent projections of the rat lateral habenular nucleus revealed by the PHA-L anterograde tracing method. Brain Res., 441, 319-330.

Balcita-Pedicino, J.J., Omelchenko, N., Bell, R. \& Sesack, S.R. (2011) The inhibitory influence of the lateral habenula on midbrain dopamine cells: ultrastructural evidence for indirect mediation via the rostromedial mesopontine tegmental nucleus. J. Comp. Neurol., 519, 1143-1164.

Barrot, M., Sesack, S.R., Georges, F., Pistis, M., Hong, S. \& Jhou, T.C (2012) Braking dopamine systems: a new GABA master structure for mesolimbic and nigrostriatal functions. J. Neurosci., 32, 14094-14101.

Bourdy, R. \& Barrot, M. (2012) A new control center for dopaminergic systems: pulling: the VTA by the tail. Trends. Neurosci., 35, 681-690.

Brinschwitz, K., Dittgen, A., Madai, V.I., Lommel, R., Geisler, S. \& Veh, R.W. (2010) Glutamatergic axons from the lateral habenula mainly terminate on GABAergic neurons of the ventral midbrain. Neuroscience, $\mathbf{1 6 8}$, 463-476.

Bromberg-Martin, E.S. \& Hikosaka, O. (2011) Lateral habenula neurons signal errors in the prediction of reward information. Nat. Neurosci., 14, 1209-1216.

Bromberg-Martin, E.S., Matsumoto, M. \& Hikosaka, O. (2010a) Distinct tonic and phasic anticipatory activity in lateral habenula and dopamine neurons. Neuron, 67, 144-155.

Bromberg-Martin, E.S., Matsumoto, M. \& Hikosaka, O. (2010b) Dopamine in motivational control: rewarding, aversive, and alerting. Neuron, 68, 815-834.

Deroche-Gamonet, V., Belin, D. \& Piazza, P.V. (2004) Evidence for addiction-like behavior in the rat. Science, 305, 1014-1017.

Erlander, M.G., Tillakaratne, N.J., Feldblum, S., Patel, N. \& Tobin, A.J. (1991) Two genes encode distinct glutamate decarboxylases. Neuron, 7 91-100.

Everitt, B.J. \& Robbins, T.W. (2016) Drug addiction: updating actions to habits to compulsions ten years on. Annu. Rev. Psychol., 67, 23-50.

Feil, J., Sheppard, D., Fitzgerald, P.B., Yucel, M., Lubman, D.I. \& Bradshaw, J.L. (2010) Addiction, compulsive drug seeking, and the role of frontostriatal mechanisms in regulating inhibitory control. Neurosci. Biobehav. R., 35, 248-275.

Gao, P., de Munck, J.C., Limpens, J.H.W., Vanderschuren, L.J.M.J. \& Voorn, P. (2017) A neuronal activation correlate in striatum and prefrontal cortex of prolonged cocaine intake. Brain Struct. Funct., 222, 3453-3475. https://doi.org/10.1007/s00429-017-1412-4 [Epub ahead of print].

Geisler, S., Andres, K.H. \& Veh, R.W. (2003) Morphologic and cytochemical criteria for the identification and delineation of individual subnuclei within the lateral habenular complex of the rat. J. Comp. Neurol., 458, 78-97.

Goldstein, R.Z. \& Volkow, N.D. (2011) Dysfunction of the prefrontal cortex in addiction: neuroimaging findings and clinical implications. Nat. Rev. Neurosci., 12, 652-669.

Goncalves, L., Sego, C. \& Metzger, M. (2012) Differential projections from the lateral habenula to the rostromedial tegmental nucleus and ventral tegmental area in the rat. J. Comp. Neurol., 520, 1278-1300.

Hearing, M.C., Zink, A.N. \& Wickman, K. (2012) Cocaine-induced adaptations in metabotropic inhibitory signaling in the mesocorticolimbic system. Rev. Neuroscience, 23, 325-351.

Herkenham, M. \& Nauta, W.J. (1977) Afferent connections of the habenular nuclei in the rat. A horseradish peroxidase study, with a note on the fiberof-passage problem. J. Comp. Neurol., 173, 123-146.

Herkenham, M. \& Nauta, W.J. (1979) Efferent connections of the habenular nuclei in the rat. J. Comp. Neurol., 187, 19-47.

Hikosaka, O., Sesack, S.R., Lecourtier, L. \& Shepard, P.D. (2008) Habenula: crossroad between the basal ganglia and the limbic system. J. Neurosci. 28, $11825-11829$

Jean-Richard-Dit-Bressel, P. \& McNally, G.P. (2014) The role of the lateral habenula in punishment. PLOS ONE, 9, e111699.

Jentsch, J.D. \& Taylor, J.R. (1999) Impulsivity resulting from frontostriatal dysfunction in drug abuse: implications for the control of behavior by reward-related stimuli. Psychopharmacology, 146, 373-390.

Jhou, T.C., Fields, H.L., Baxter, M.G., Saper, C.B. \& Holland, P.C. (2009a) The rostromedial tegmental nucleus (RMTg), a GABAergic afferent to midbrain dopamine neurons, encodes aversive stimuli and inhibits motor responses. Neuron, 61, 786-800. 
Jhou, T.C., Geisler, S., Marinelli, M., Degarmo, B.A. \& Zahm, D.S. (2009b) The mesopontine rostromedial tegmental nucleus: a structure targeted by the lateral habenula that projects to the ventral tegmental area of Tsai and substantia nigra compacta. J. Comp. Neurol., 513, 566-596.

Jhou, T.C., Good, C.H., Rowley, C.S., Xu, S.P., Wang, H., Burnham, N.W., Hoffman, A.F., Lupica, C.R. et al. (2013) Cocaine drives aversive conditioning via delayed activation of dopamine-responsive habenular and midbrain pathways. J. Neurosci., 33, 7501-7512.

Ji, H. \& Shepard, P.D. (2007) Lateral habenula stimulation inhibits rat midbrain dopamine neurons through a $\operatorname{GABA}(\mathrm{A})$ receptor-mediated mechanism. J. Neurosci., 27, 6923-6930.

Jonkman, S., Pelloux, Y. \& Everitt, B.J. (2012) Drug intake is sufficient, but conditioning is not necessary for the emergence of compulsive cocaine seeking after extended self-administration. Neuropsychopharmacology, 37, 1612-1619.

Kaufling, J., Veinante, P., Pawlowski, S.A., Freund-Mercier, M.J. \& Barrot, M. (2009) Afferents to the GABAergic tail of the ventral tegmental area in the rat. J. Comp. Neurol., 513, 597-621.

Koob, G.F. (2013) Negative reinforcement in drug addiction: the darkness within. Curr. Opin. Neurobiol., 23, 559-563.

Koob, G.F. \& Volkow, N.D. (2010) Neurocircuitry of addiction. Neuropsychopharmacology, 35, 217-238.

Kowski, A.B., Geisler, S., Krauss, M. \& Veh, R.W. (2008) Differential projections from subfiekds in the lateral preoptic area to the lateral habenular complex of the rat. J. Comp. Neurol., 507, 1465-1478.

Kowski, A.B., Veh, R.W. \& Weiss, T. (2009) Dopaminergic activation excites rat lateral habenular neurons in vivo. Neuroscience, 161, 11541165.

Lammel, S., Lim, B.K., Ran, C., Huang, K.W., Betley, M.J., Tye, K.M., Deisseroth, K. \& Malenka, R.C. (2012) Input-specific control of reward and aversion in the ventral tegmental area. Nature, 491, 212-217.

Lax, E., Friedman, A., Croitoro, O., Sudai, E., Ben-Moshe, H., Redlus, L., Sasson, E., Katzir, T. et al. (2013) Neurodegeneration of lateral habenula efferent fibers after intermittent cocaine administration: implications for deep brain stimulation. Neuropharmacology, 75, 246-254.

Lecca, S., Meye, F.J. \& Mameli, M. (2014) The lateral habenula in addiction and depression: an anatomical, synaptic and behavioral overview. Eur. J. Neurosci., 39, 1170-1178.

Lecourtier, L. \& Kelly, P.H. (2007) A conductor hidden in the orchestra? Role of the habenular complex in monoamine transmission and cognition. Neurosci. Biobehav. R., 31, 658-672.

Li, B., Piriz, J., Mirrione, M., Chung, C., Proulx, C.D., Schulz, D., Henn, F. \& Malinow, R. (2011) Synaptic potentiation onto habenula neurons in the learned helplessness model of depression. Nature, 470, 535-539.

Limpens, J.H.W., Schut, E.H.S., Voorn, P. \& Vanderschuren, L.J.M.J. (2014) Using conditioned suppression to investigate compulsive drug seeking in rats. Drug Alcohol Depen., 142, 314-324.

Mahler, S.V. \& Aston-Jones, G. (2012) Fos activation of selective afferents to ventral tegmental area during cue-induced reinstatement of cocaine seeking in rats. J. Neurosci., 32, 13309-13325.

Maroteaux, M. \& Mameli, M. (2012) Cocaine evokes projection-specific synaptic plasticity of lateral habenula neurons. J. Neurosci., 32, 1264112646.

Matsumoto, M. \& Hikosaka, O. (2007) Lateral habenula as a source of negative reward signals in dopamine neurons. Nature, 447, 1111-1115.

Matsumoto, M. \& Hikosaka, O. (2009) Representation of negative motivational value in the primate lateral habenula. Nat. Neurosci., 12, 7784.

Meshul, C.K., Noguchi, K., Emre, N. \& Ellison, G. (1998) Cocaine-induced changes in glutamate and GABA immunolabeling within rat habenula and nucleus accumbens. Synapse, 30, 211-220.

Meye, F.J., Valentinova, K., Lecca, S., Marion-Poll, L., Maroteaux, M.J., Musardo, S., Moutkine, I., Gardoni, F. et al. (2015) Cocaine-evoked negative symptoms require AMPA receptor trafficking in the lateral habenula. Nat. Neurosci., 18, 376-378.
Neumann, P.A., Ishikawa, M., Otaka, M., Huang, Y.H., Schlüter, O.M. \& Dong, Y. (2014) Increased excitability of lateral habenula neurons in adolescent rats following cocaine self-administration. Int. J. Neuropsychop., 18, $1-10$.

Oliva, I. \& Wanat, M.J. (2016) Ventral tegmental area afferents and drugdependent behaviors. Front. Psychiat., 7, https://doi.org/10.3389. [Epub ahead of print].

Omelchenko, N., Bell, R. \& Sesack, S.R. (2009) Lateral habenula projections to dopamine and GABA neurons in the rat ventral tegmental area. Eur. J. Neurosci., 30, 1239-1250.

Pelloux, Y., Everitt, B.J. \& Dickinson, A. (2007) Compulsive drug seeking by rats under punishment: effects of drug taking history. Psychopharmacology, 194, 127-137.

Petzel, A., Bernard, R., Poller, W.C. \& Veh, R.W. (2017) Anterior and posterior parts of the rat ventral tegmental area and the rostromedoal tegmental nucleus receive topographically distinct afferents from the lateral complex. J. Comp. Neurol., 525, 2310-2327. https://doi.org/10.1002/cne. 24200 [Epub ahead of print].

Piazza, P.V. \& Deroche-Gamonet, V. (2013) A multistep general theory of transition to addiction. Psychopharmacology, 229, 387-413.

Proulx, C.D., Hikosaka, O. \& Malinow, R. (2014) Reward processing by the lateral habenula in normal and depressive behaviors. Nat. Neurosci., 17, 1146-1152.

Quina, L.A., Tempest, L., Ng, L., Harris, J.A., Ferguson, S., Jhou, T.C. \& Turner, E.E. (2015) Efferent pathways of the mouse lateral habenula. $J$. Comp. Neurol., 523, 32-60.

Robinson, T.E. \& Berridge, K.C. (2003) Addiction. Annu. Rev. Psychol., 54, 25-53.

Sego, C., Goncalves, L., Lima, L., Furigo, I.C., Donato, J. Jr \& Metzger, M. (2014) Lateral habenula and the rostromedial tegmental nucleus innervate neurochemically distinct subdivisions of the dorsal raphe nucleus in the rat. J. Comp. Neurol., 522, 1454-1484.

Shelton, K., Bogyo, K., Schick, T. \& Ettenberg, A. (2016) Pharmacological modulation of lateral habenular dopamine D2 receptors alters the anxiogenic response to cocaine in a runway model of drug self-administration. Behav. Brain Res., 310, 42-50.

Vanderschuren, L.J.M.J. \& Ahmed, S.H. (2013) Animal studies of addictive behavior. CSH Perspect. Med., 3, a011932.

Vanderschuren, L.J.M.J. \& Everitt, B.J. (2004) Drug seeking becomes compulsive after prolonged cocaine self-administration. Science, 305, 1017-1019.

Vanderschuren, L.J.M.J., Minnaard, A.M., Smeets, J.A.S. \& Lesscher, H.M.B. (2017) Punishment models of addictive behavior. Curr. Opin. Behav. Sci., 13, 77-84.

Veeneman, M.M.J., Broekhoven, M.H., Damsteegt, R. \& Vanderschuren, L.J.M.J. (2012) Distinct contributions of dopamine in the dorsolateral striatum and nucleus accumbens shell to the reinforcing properties of cocaine. Neuropsychopharmacology, 37, 487-498.

Volkow, N.D. \& Morales, M. (2015) The brain on drugs: from reward to addiction. Cell, 162, 712-725.

Wirtshafter, D., Asin, K.E. \& Pitzer, M.R. (1994) Dopamine agonists and stress produce different patterns of Fos-like immunoreactivity in the lateral habenula. Brain Res., 633, 21-26.

Zahm, D.S., Becker, M.L., Freiman, A.J., Strauch, S., Degarmo, B., Geisler, S., Meredith, G.E. \& Marinelli, M. (2010) Fos after single and repeated self-administration of cocaine and saline in the rat: emphasis on the Basal forebrain and recalibration of expression. Neuropsychopharmacology, 35, $445-463$.

Zapata, A., Huang, E.K. \& Lupica, C.R. (2017) Lateral habenula involvement in impulsive cocaine seeking. Neurosychopharmacology, 42, 1103-1112.

Zhang, F., Zhou, W., Liu, H., Zhu, H., Tang, S., Lai, M. \& Yang, G. (2005) Increased c-Fos expression in the medial part of the lateral habenula during cue-evoked heroin-seeking in rats. Neurosci. Lett., 386, 133-137.

Zuo, W., Chen, L., Wang, L. \& Ye, J.H. (2013) Cocaine facilitates glutamatergic transmission and activates lateral habenular neurons. Neuropharmacology, 70, 180-189. 